\title{
Cone-beam computed tomography of mandibular foramen and lingula for mandibular anesthesia
}

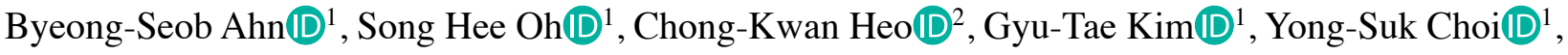 \\ Eui-Hwan Hwang(D) ${ }^{1}$ \\ ${ }^{1}$ Department of Oral and Maxillofacial Radiology, School of Dentistry, Kyung Hee University, Seoul, Korea \\ ${ }^{2}$ School of Dentistry, Kyung Hee University, Seoul, Korea
}

\section{ABSTRACT}

\begin{abstract}
Purpose: The positions of the mandibular foramen $(\mathrm{MnF})$ and the lingula affect the success rate of inferior alveolar nerve block. The objective of this study was to investigate aspects of the $\mathrm{MnF}$ and the lingula relevant for mandibular block anesthesia using cone-beam computed tomography (CBCT).

Materials and Methods: Fifty CBCT scans were collected from a picture archiving and communications system. All scans were taken using an Alphard Vega 3030 (Asahi Roentgen Co. Ltd., Kyoto, Japan). Fifty-eight MnFs of 30 subjects were included in the study. The position of the $\mathrm{MnF}$, the size of the MnF, the position of the lingula, the size of the lingula, and the shape of the lingula were measured and recorded. All data were statistically analyzed at a significance level of $P<0.05$.

Results: The position of $\mathrm{MnF}$ was $0.1 \mathrm{~mm}$ and $0.8 \mathrm{~mm}$ below the occlusal plane in males and females, respectively. The horizontal position of the $\mathrm{MnF}$ was slightly anterior to the center of the ramus in males and in the center in females $(P<0.05)$. The vertical position of the $\mathrm{MnF}$ was lower in females than in males $(P<0.05)$. The $\mathrm{MnF}$ was an oval shape with a longer anteroposterior dimension. The height of the lingula was $9.3 \mathrm{~mm}$ in males and $8.2 \mathrm{~mm}$ in females. The nodular type was the most common shape of the lingula, followed by the triangular, truncated, and assimilated types.

Conclusion: CBCT provided useful information about the $\mathrm{MnF}$ and lingula. This information could improve the success rate of mandibular blocks. (Imaging Sci Dent 2020; 50: 125-32)
\end{abstract}

KEY WORDS: Mandible; Anatomy, Cross-Sectional; Nerve Block; Cone-Beam Computed Tomography

\section{Introduction}

The inferior alveolar nerve (IAN) is the largest branch of the mandibular nerve and gives off the mylohyoid, dental, incisive, and mental branches. The IAN branches off the mandibular nerve and enters the mandible through the mandibular foramen $(\mathrm{MnF})$, which is located on the medial surface of the ramus of the jaw. ${ }^{1}$

The lingula is a structure located near the $\mathrm{MnF}$ on the medial side of the mandibular ramus. Tuli et al. ${ }^{2}$ classified the shape of the lingula into 4 types: triangular, truncated,

Received December 24, 2018; Revised March 28, 2020; Accepted April 12, 2020 *Correspondence to : Prof. Eui-Hwan Hwang

Department of Oral and Maxillofacial Radiology, School of Dentistry, Kyung Hee University, 26 Kyungheedaero Dongdaemun-gu, Seoul 02447, Korea

Tel) 82-2-958-9406, E-mail) hehan@khu.ac.kr nodular, and assimilated. It is a landmark for mandibular nerve block anesthesia and in mandibular orthognathic surgery such as sagittal ramus osteotomy.,

IAN block is an anesthesia technique for the mandible. Infiltration is effective in the anterior area; however, it is insufficient in the posterior region of the mandible because of differences in the cortication thickness. In contrast, infiltration is sufficient for most cases in the maxilla due to its thinner cortical plate and lower density compared to the mandible. ${ }^{5}$

However, IAN block is challenging and does not have a high success rate. ${ }^{6,7}$ This technique is often unsuccessful even when performed by dentists who have several years of experience. The failure rate of IAN block is especially high in the anterior mandibular area. Furthermore, its com- 
plications include direct nerve penetration, hematoma, and anesthetic toxicity. ${ }^{6}$ When IAN block fails, an alternative anesthetic technique, such as high-level block of the IAN, may be effective. The Gow-Gates technique or the Vazirani-Akinosi (or Akinosi-Vazirani) technique could be the second choice for high-level nerve block.,

Soft tissues obscure bony landmarks for IAN block procedures. Instead, the only reliable indicators in the IAN block technique are soft tissue landmarks in the mouth and the tactile sense of the operator. The dentist must inject the anesthetic in the pterygomandibular space near the lingula and the $\mathrm{MnF}$ of the mandible. ${ }^{8}$ Variation in the location of the $\mathrm{MnF}$ and the lingula contribute to the low success rate. In addition, bifid mandibular canals can cause anesthesia to fail. ${ }^{8}$ Another cause of anesthetic failure is the innervation of mandibular teeth by other nerves. Furthermore, the patient's psychological state can also affect the success rate of anesthesia.?
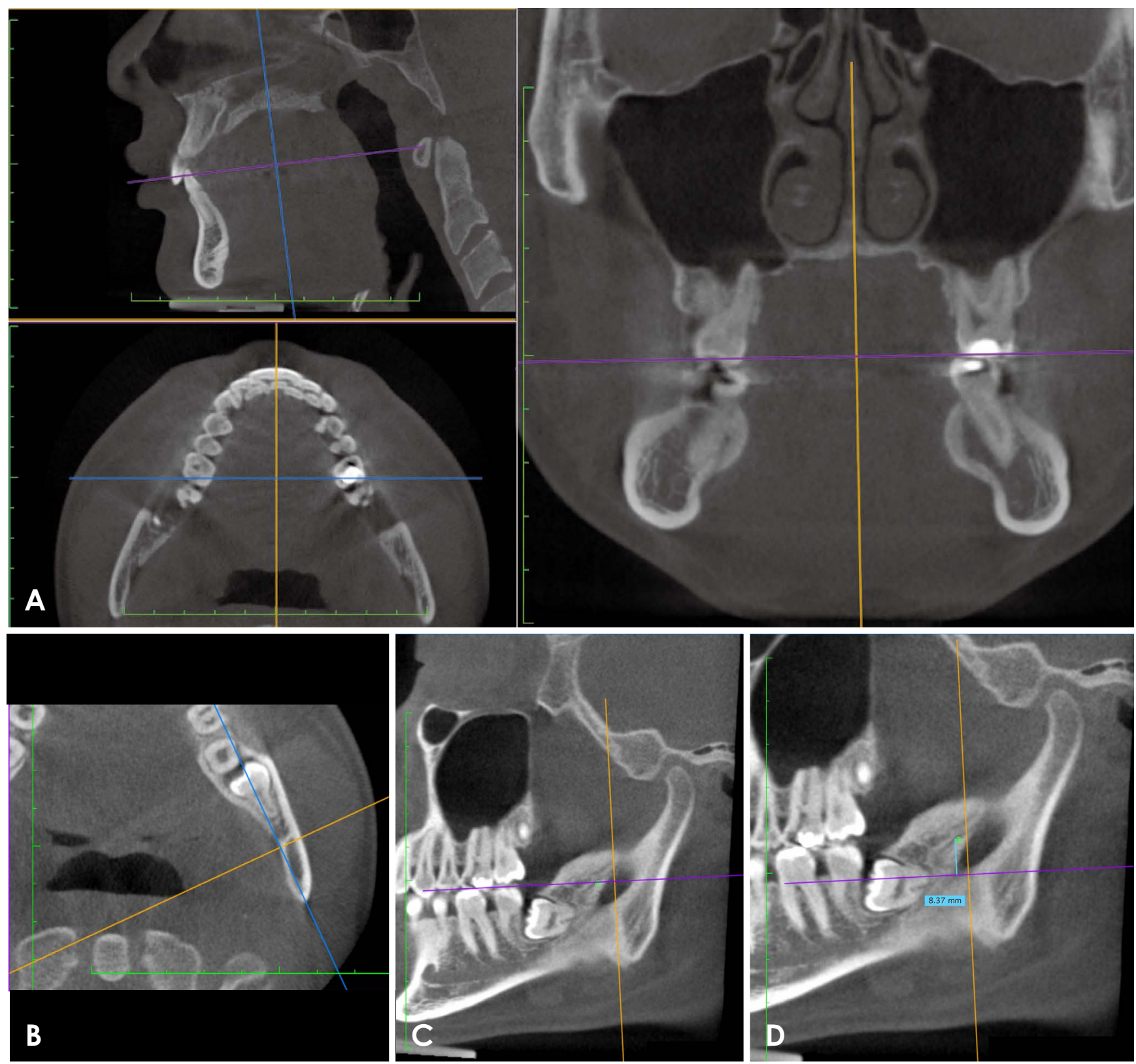

Fig. 1. Measurement of the distance between the occlusal plane and the mandibular foramen. A. Rotation of the axial plane to the occlusal plane. B. The center of the 3 planes is moved to the center of the mandibular foramen. The sagittal plane is aligned with the line passing from the center point of the ramus anterior border to the posterior border of the mandible. C. A reference point (green double circle) is marked on the axial plane line. D. The axial plane is moved to where the cortication of the mandibular foramen is completed. The distance from the reference point to the axial plane is measured. 
Several studies have investigated the anatomy of the MnF using dry skulls, ${ }^{2,3}$ plain radiography, ${ }^{9,10}$ or conventional computed tomography $(\mathrm{CT}) .{ }^{12}$ Plain radiographs, even cephalometric radiographs, usually show distorted images, while panoramic radiographs provide various magnifications of the maxilla and the mandible. In contrast, cone-beam CT (CBCT), which is commonly used in dental clinics, can produce highly accurate images ${ }^{14}$ and provide precise locations of anatomical structures.

The position of the $\mathrm{MnF}$ and the shape of the lingula are expected to affect the success rate of IAN block. However, few CBCT studies of these structures have been conducted. Therefore, the purpose of this study was to provide anatomical information on the $\mathrm{MnF}$ and lingula relevant for IAN block using CBCT.

\section{Materials and Methods}

In this study, CBCT data of 50 patients were selected randomly from the picture archiving and communication system of Kyung Hee University Dental Hospital from a 6-month period. The scans had been taken for orthodontic treatments, orthognathic surgery, and temporomandibular disorder using an Alphard Vega 3030 (Asahi Roentgen Co. Ltd., Kyoto, Japan) with parameters of $80 \mathrm{kVp}, 10 \mathrm{~mA}$, and 13.3 seconds. The exclusion criteria were an asymmetric mandibular shape, a history of mandibular fracture, and a history of mandibular surgery. Thirty of the 50 initial patients were included in the study. Two MnFs were excluded from the measurements because they were not well observed due to movement artifacts. Thus, in total, $58 \mathrm{MnFs}$ and lingulae were studied in this study.

The image processing software OsiriX (v. 4.0; http://www. osirix.com) on MacOS X (version 10.8; Apple, Inc., Cupertino, CA, USA) was used to study the MnF and its surrounding structures. All measurements were made on multi-planar reconstruction (MPR) with maximum intensity projection (MIP). Observations of the shape of the lingula were made on volume-rendering mode using the InVivoDental application (v. 5.2; Anatomage Inc., San Jose, CA, USA).

To measure the distance between the occlusal plane and the MnF, the axial plane was adjusted to the occlusal plane (Fig. 1A). The center of the 3 planes was moved to the center of the MnF. The sagittal plane line was then rotated on the axial plane until it met the line passing from the midpoint of the anterior border or the distal side of the second molar area to the posterior border of the mandible (Fig. 1B). The point was marked on the axial plane line as
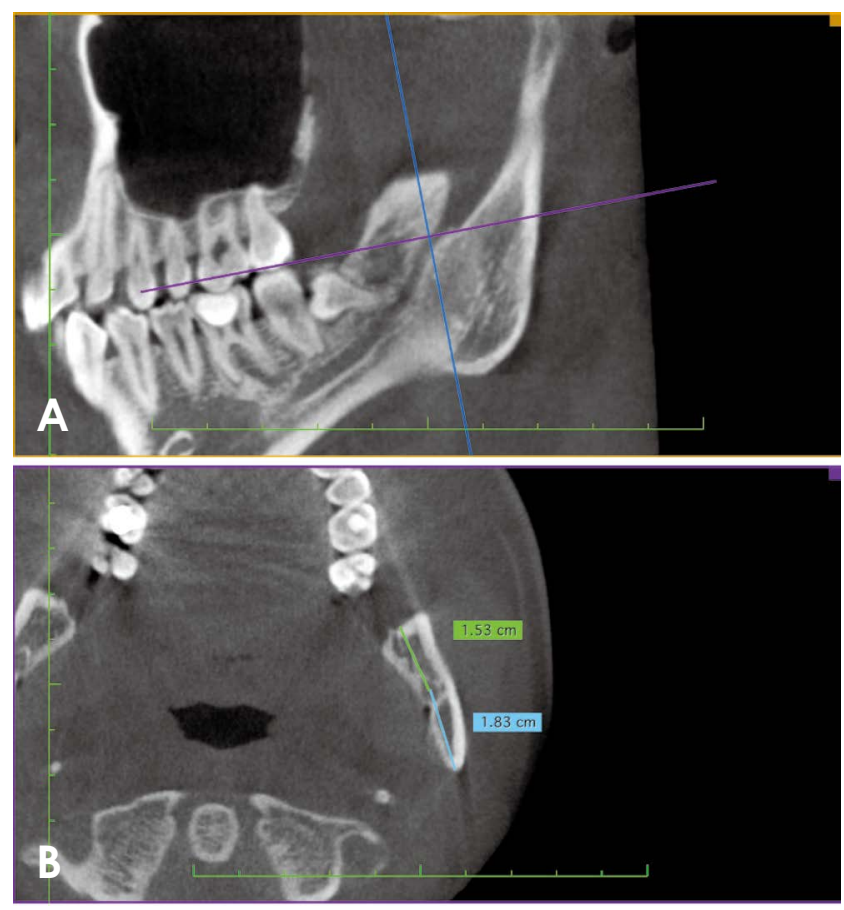

Fig. 2. Distance between the anterior and posterior borders and the mandibular foramen. A. The center of the 3 planes is positioned at the center of the mandibular foramen. B. Anterior and posterior distances are measured from the center of the mandibular foramen.

a reference point (Fig. 1C). The axial plane line was moved to where the cortication of the $\mathrm{MnF}$ was completed. A measurement was made between the reference point and the axial plane on the rotated sagittal plane (Fig. 1D). A positive value indicated that the MnF was above the occlusal plane, and a negative value meant that it was below the occlusal plane.

The anterior border and the posterior border of the ramus were confirmed on the same plane as the MnF. Specifically, the distances from the center of the $\mathrm{MnF}$ to each anterior and posterior border were measured on the axial plane (Fig. 2).

To measure the distances between the sigmoid notch and the $\mathrm{MnF}$ and between the gonion and the MnF, the thickness was adjusted and MIP-reconstructed (Fig. 3A). The thickness was set to sufficient to cover the entire thickness of the ramus in order to visualize the sigmoid notch and the gonion (Figs. 3B and C). The distances from the center of the $\mathrm{MnF}$ to the sigmoid notch and to the gonion were measured.

The anteroposterior and mediolateral diameters of the $\mathrm{MnF}$ were measured (Fig. 4). The axial plane line was rotated until the frontal plane line aligned with the long axis of the mandibular canal (Fig. 4A). If this procedure had not been done, the anteroposterior diameter would have been longer than the real size of the MnF. 

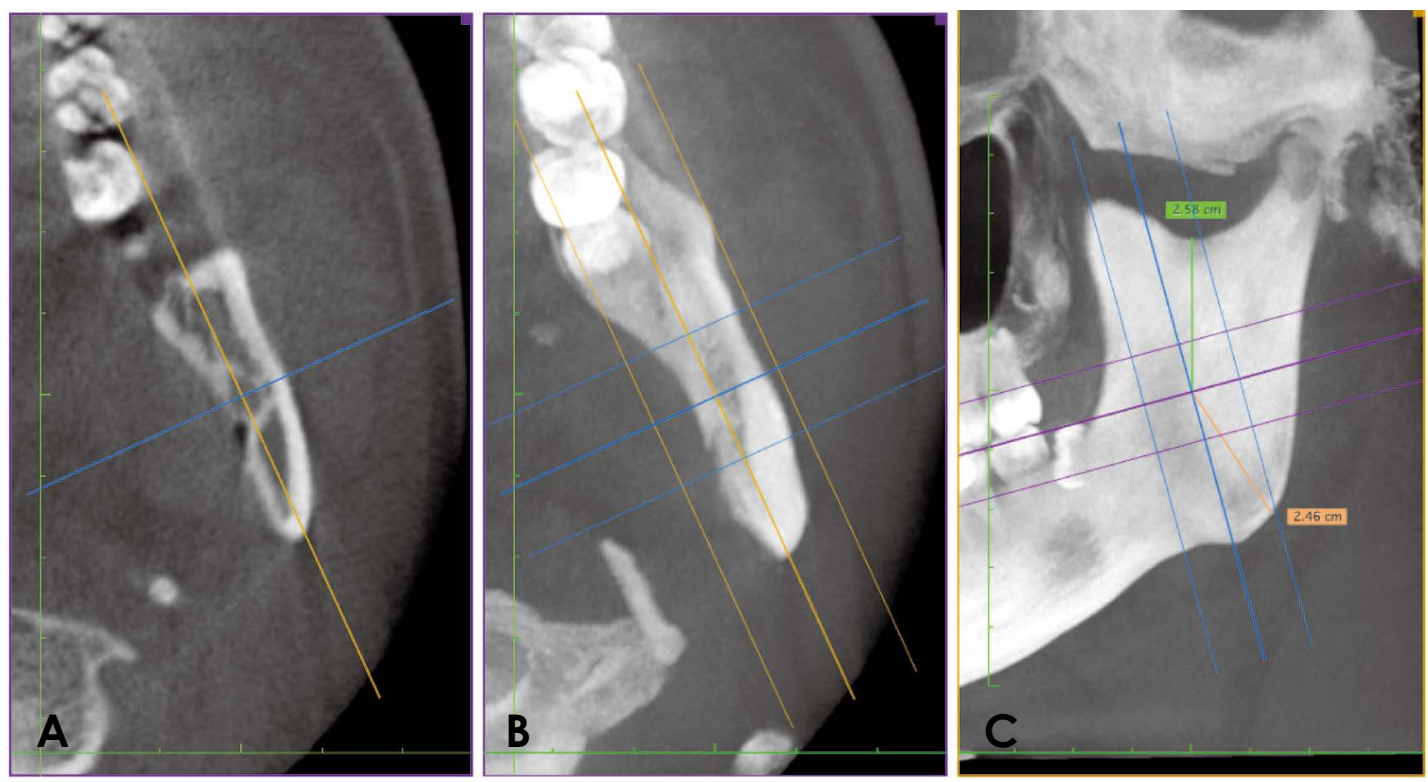

Fig. 3. Distance between the sigmoid notch and the mandibular foramen. A. The center of the 3 planes is positioned at the center of the mandibular foramen. B. The thickness is applied using the maximum intensity projection to visualize the sigmoid notch and the gonion. C. The distances from the sigmoid notch and the gonion to the center of the mandibular foramen are measured.
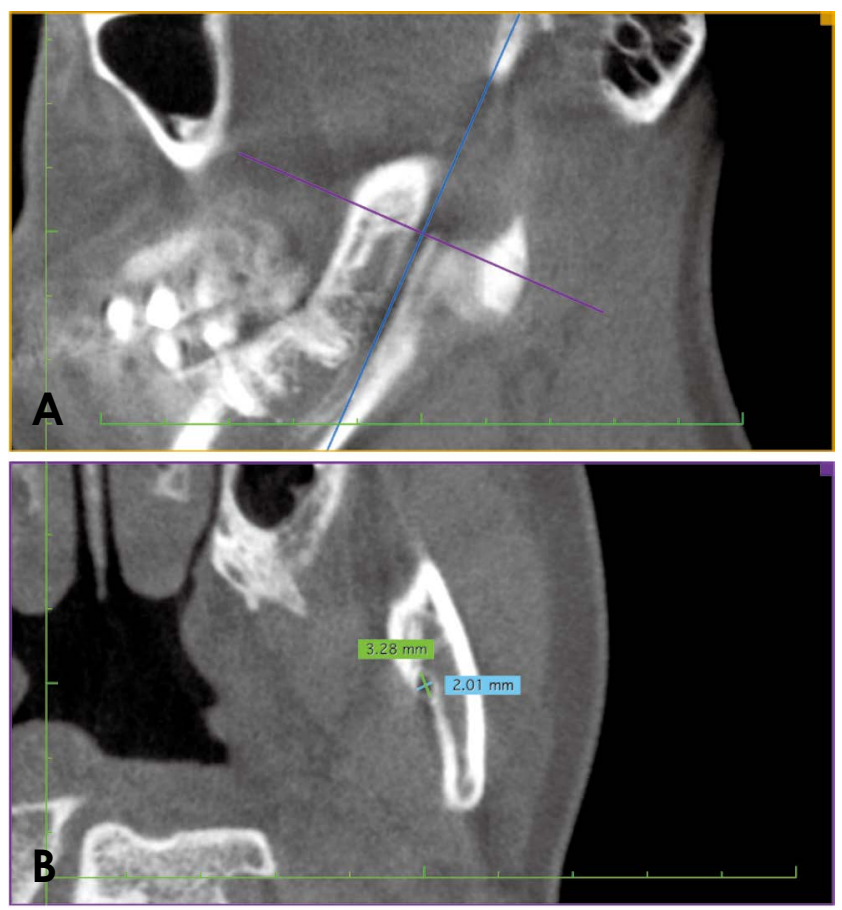

Fig. 4. Anteroposterior and mediolateral diameters of the mandibular foramen. A. The axial plane line is rotated until the frontal plane line aligned with the long axis of the mandibular canal. B. Diameters are measured anteroposteriorly and mediolaterally.

For the lingula size, vertical length was measured from the center of the MnF to the most protruding point of the lingula (Fig. 5). For this measurement, the axial plane line
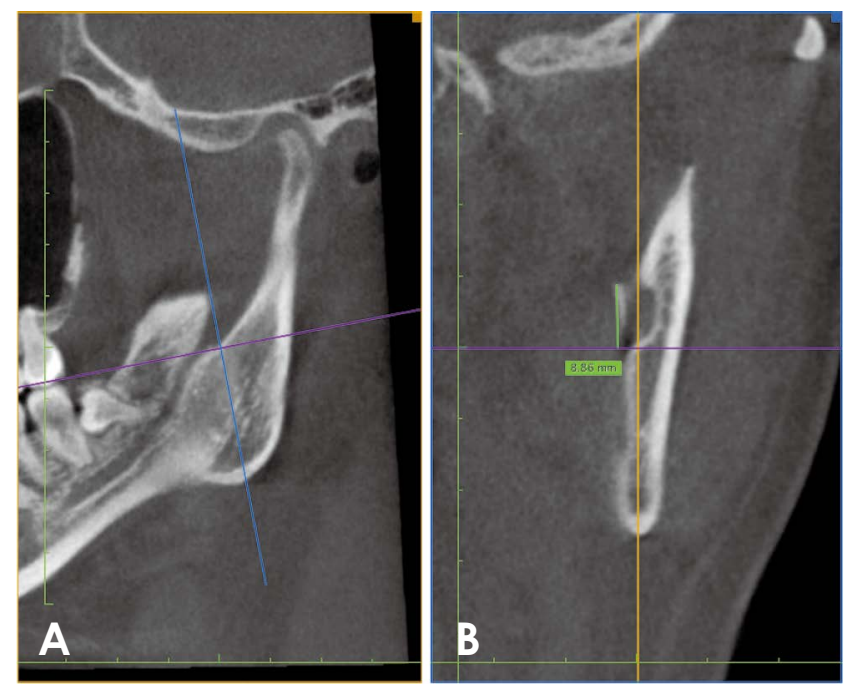

Fig. 5. Size of the lingula. A. The center of the 3 planes is on the center of the mandibular foramen. B. The frontal plane line is moved to where the tip of the lingula could be confirmed. The vertical length is measured between the tip of the lingula and the center of the mandibular foramen.

was rotated to the position parallel to the occlusal plane. The frontal plane line was moved to visualize the ligula. The vertical distance from the axial line to the tip of the lingula was measured (Fig. 5B).

The shape of the lingula was observed on 3-dimensional (3-D) volume rendering, and the shapes were classified according to the system proposed by Tuli et al. ${ }^{2}$ as triangular, 

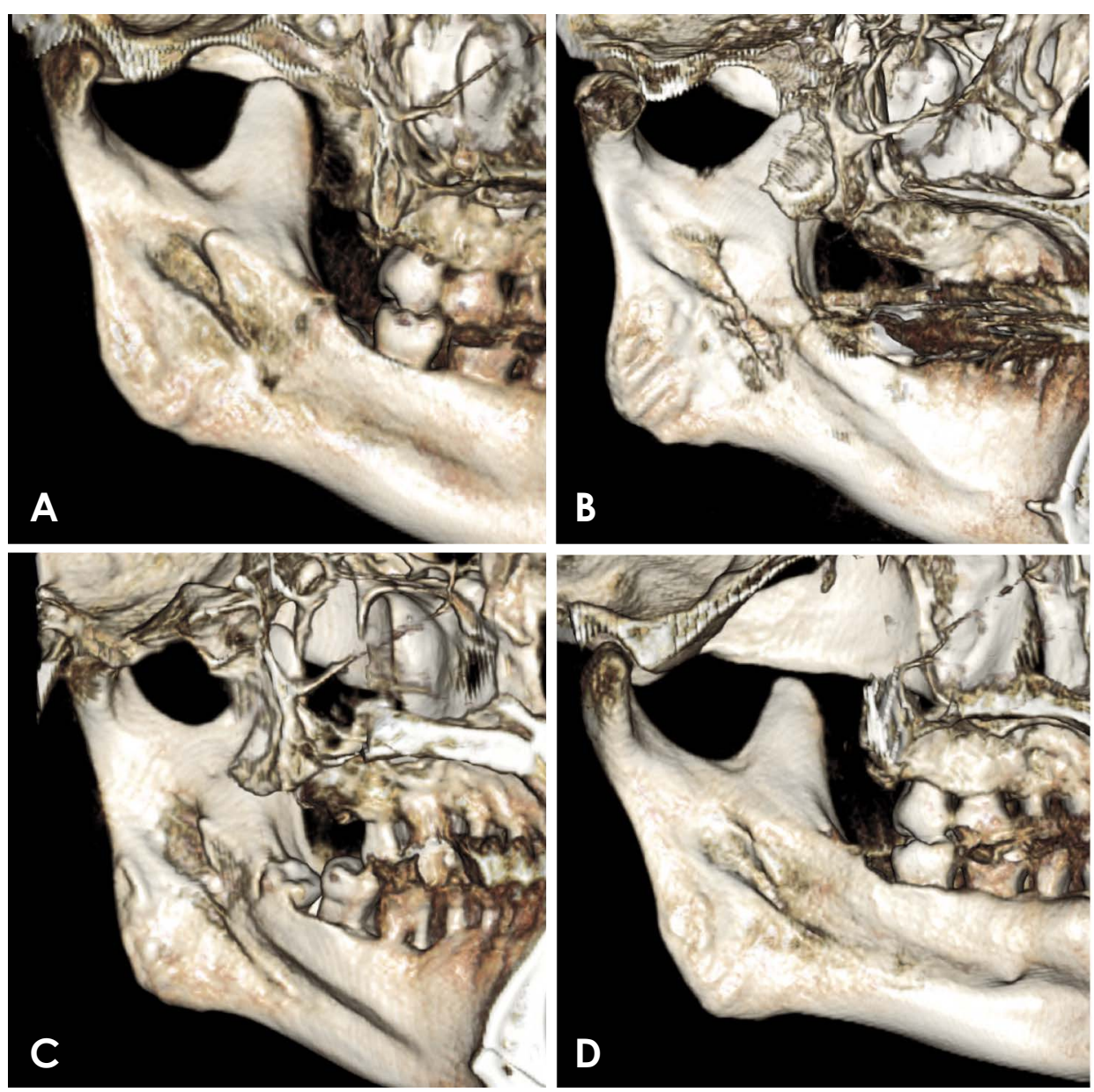

Fig. 6. Shapes of the lingula. A. Triangular type. B. Truncated type. C. Nodular type. D. Assimilated type of the lingula. The shapes are observed in 3-dimensional volume-rendering mode. truncated, nodular, or assimilated (Fig. 6).

All measurements were statistically analyzed using SPSS version 20.0 (IBM Corp., Armonk, NY, USA). Mixed repeated-measures analysis of variance was used to evaluate the significance of differences in each variables according to sex and side. The threshold for statistical significance of between-group differences was set at $P<0.05$.

\section{Results}

All results were expressed as mean \pm standard deviation. The distance between the occlusal plane and the MnF was $-0.1 \pm 4.6 \mathrm{~mm}$ in males, while it was $-0.8 \pm 3.1 \mathrm{~mm}$ in females (Table 1). The distance between the anterior border of the mandibular ramus and the $\mathrm{MnF}$ was $15.3 \pm 2.7$ $\mathrm{mm}$ in males and $15.7 \pm 2.6 \mathrm{~mm}$ in females. The distance between the posterior border of the mandibular ramus and the $\mathrm{MnF}$ was $19.1 \pm 2.2 \mathrm{~mm}$ in males and $16.9 \pm 1.5 \mathrm{~mm}$ in females, which was a statistically significant difference $(P<0.05)$ (Table 2). The average distance between the sigmoid notch and the MnF was $23.9 \pm 3.6 \mathrm{~mm}$ in males and
Table 1. Distance between the occlusal plane and mandibular foramen

\begin{tabular}{llcc}
\hline & & Distance $(\mathrm{mm})$ & Number \\
\hline Right & Male & $-0.5 \pm 5.1$ & 15 \\
& Female & $-0.3 \pm 2.9$ & 14 \\
\multirow{2}{*}{ Left } & Male & $0.4 \pm 4.4$ & 15 \\
& Female & $-1.3 \pm 3.2$ & 14 \\
\hline
\end{tabular}

A minus sign (-) means that the mandibular foramen is below the occlusal plane.

$21.8 \pm 4.3 \mathrm{~mm}$ in females (Table 3 ). The distance between the gonion and the $\mathrm{MnF}$ was $22.3 \pm 4.5 \mathrm{~mm}$ in males and $19.6 \pm 2.5 \mathrm{~mm}$ in females. This distance showed statistically significant differences according to sex and side of the mouth $(P<0.05)$ (Table 3$)$. The anteroposterior diameter of the mandibular foramen was $3.2 \pm 0.7 \mathrm{~mm}$, while the mediolateral diameter was $2.7 \pm 0.6 \mathrm{~mm}$ (Table 4 ). The distance between the lingula and $\mathrm{MnF}$ was $9.3 \pm 2.4 \mathrm{~mm}$ in males and $8.2 \pm 2.6 \mathrm{~mm}$ in females (Table 5).

The most common shape of the lingula was the nodular type $(32.8 \%)$, followed by the triangular type $(31.0 \%)$, the 
truncated type (25.9\%), and the assimilated type (10.3\%). In males, the triangular type was the most common (33.3\%), followed by the nodular type (20.0\%), the truncated type $(30.0 \%)$, and the assimilated type (16.7\%). In females, the nodular type (35.7\%) was the most common, followed by the truncated type (32.1\%), the triangular type (28.6\%), and the assimilated type (3.6\%) (Table 6).

\section{Discussion}

The position of the $\mathrm{MnF}$ and the position and shape of the lingula affect the success rate of IAN block. Previous

Table 2. Distance between the anterior and posterior borders of the mandibular ramus and mandibular foramen $(\mathrm{mm})$

\begin{tabular}{llccc}
\hline & & Anterior border & Posterior border* & Number \\
\hline Right & Male & $15.7 \pm 2.6$ & $19.3 \pm 2.2$ & 15 \\
& Female & $15.3 \pm 2.3$ & $17.1 \pm 1.4$ & 14 \\
Left & Male & $15.2 \pm 2.5$ & $19.0 \pm 2.2$ & 15 \\
& Female & $15.9 \pm 2.4$ & $16.9 \pm 1.6$ & 14 \\
\hline
\end{tabular}

$*: P<0.05$ between males and females

Table 3. Distance from the mandibular foramen to sigmoid notch and gonion $(\mathrm{mm})$

\begin{tabular}{llccc}
\hline & & Sigmoid notch & Gonion* & Number \\
\hline Right & Male & $24.0 \pm 4.0$ & $21.5 \pm 4.6$ & 15 \\
& Female & $22.1 \pm 3.7$ & $19.3 \pm 2.6$ & 14 \\
\multirow{2}{*}{ Left } & Male & $23.8 \pm 3.3$ & $23.1 \pm 4.5$ & 15 \\
& Female & $21.7 \pm 5.0$ & $19.9 \pm 2.4$ & 14 \\
\hline
\end{tabular}

*: $P<0.05$ between males and females and between both sides $(P=0.029)$ studies have investigated the location of the lingula and/ or $\mathrm{MnF}^{2,3,9,10}$ but no study in the literature has yet investigated the relationship between the shape of the lingula and the success rate of anesthesia. Nonetheless, a prior study did analyze the position of the lingula and the success rate of IAN block. ${ }^{11}$ That study argued that the lingula on panoramic radiography is not a suitable landmark for the needle insertion point for IAN block due to the distortion present on panoramic radiographs. By contrast, measurements of distance and angles on CT and CBCT correspond very closely to the actual size of the object. ${ }^{12}$

Table 4. Diameter of the mandibular foramen ( $\mathrm{mm})$

\begin{tabular}{llccc}
\hline & & $\begin{array}{c}\text { Anteroposterior } \\
\text { diameter }\end{array}$ & $\begin{array}{c}\text { Mediolateral } \\
\text { diameter* }\end{array}$ & Number \\
\hline Male & Right & $3.4 \pm 0.9$ & $2.8 \pm 0.8$ & 15 \\
& Left & $3.4 \pm 0.5$ & $2.6 \pm 0.5$ & 15 \\
\multirow{2}{*}{ Female } & Right & $3.0 \pm 0.5$ & $2.6 \pm 0.4$ & 14 \\
& Left & $3.1 \pm 0.7$ & $2.7 \pm 0.6$ & 14 \\
All & Male & $3.4 \pm 0.7$ & $2.7 \pm 0.6$ & 30 \\
& Female & $3.1 \pm 0.6$ & $2.7 \pm 0.5$ & 28 \\
\hline
\end{tabular}

*: $P<0.05$ between both sides

Table 5. Length of the lingula (mm)

\begin{tabular}{llcc}
\hline & & Length of the lingula & Number \\
\hline Right & Male & $9.4 \pm 2.6$ & 15 \\
& Female & $8.3 \pm 2.7$ & 14 \\
\multirow{2}{*}{ Left } & Male & $9.2 \pm 2.4$ & 15 \\
& Female & $8.3 \pm 2.7$ & 14 \\
\hline
\end{tabular}

Table 6. Shape of the lingula

\begin{tabular}{|c|c|c|c|c|c|c|}
\hline & & Number & Triangular type & Truncated type & Nodular type & Assimilated type \\
\hline \multirow[t]{3}{*}{ Male } & Right & 15 & $7(46.7 \%)$ & $2(13.3 \%)$ & $4(26.7 \%)$ & $2(13.3 \%)$ \\
\hline & Left & 15 & $3(20.0 \%)$ & $4(26.7 \%)$ & $5(33.3 \%)$ & $3(20.0 \%)$ \\
\hline & Both sides & 30 & $10(33.3 \%)$ & $6(20.0 \%)$ & $9(30.0 \%)$ & $5(16.7 \%)$ \\
\hline \multirow[t]{3}{*}{ Female } & Right & 14 & $3(21.4 \%)$ & $6(42.9 \%)$ & $4(28.6 \%)$ & $1(7.1 \%)$ \\
\hline & Left & 14 & $5(35.7 \%)$ & $3(21.4 \%)$ & $6(42.9 \%)$ & $0(0.0 \%)$ \\
\hline & Both sides & 28 & $8(28.6 \%)$ & $9(32.1 \%)$ & $10(35.7 \%)$ & $1(3.6 \%)$ \\
\hline \multirow[t]{3}{*}{ All } & Right & 29 & $10(34.5 \%)$ & $8(27.6 \%)$ & $8(27.6 \%)$ & $3(10.3 \%)$ \\
\hline & Left & 29 & $8(27.6 \%)$ & $7(24.1 \%)$ & $11(37.9 \%)$ & $3(10.3 \%)$ \\
\hline & Both sides & 58 & $18(31.0 \%)$ & $15(25.9 \%)$ & $19(32.8 \%)$ & $6(10.3 \%)$ \\
\hline
\end{tabular}


The diagnostic ability of CBCT has been useful to many dentists. CBCT has become increasingly common in the Republic of Korea because it has smaller voxels than conventional CT, enabling it to express fine structures. However, despite its versatility, it can only delineate calcified tissue. Although it shows the outlines of soft tissues, it lacks the ability to distinguish between soft tissues. It works at a lower tube voltage and with less tube current than CT, and the radiation dose of CBCT is lower than that of multislice CT. ${ }^{13}$ Nonetheless, depending on the instruments, the radiation dose of CBCT is several times higher than that of panoramic radiography. ${ }^{14}$

Because the MPR of CT is not an effective method for recognizing shapes with a certain thickness, we used 3-D volume rendering to observe the shape of the lingula, as it is a more effective modality for distinguishing shapes. Differences in the voxel size of CT (or CBCT) and the use of different volume rendering software can affect the results, which may explain discrepancies between our findings and those of other studies.

The anteroposterior and mediolateral diameters of the $\mathrm{MnF}$ in this study were $3.2 \pm 0.7 \mathrm{~mm}$ and $2.7 \pm 0.6 \mathrm{~mm}$, respectively; in contrast, Jansisyanont et al. ${ }^{3}$ reported that the mean anteroposterior diameter of the $\mathrm{MnF}$ was $4.7 \pm 0.8$ $\mathrm{mm}$. This discrepancy may reflect racial differences.

Tuli et al. ${ }^{2}$ reported that the most common shape of the lingula was the triangular type (68.5\%) In a study of Indian adult mandibles, followed by the truncated type (15.8\%), the nodular type (10.9\%), and the assimilated type. Kositbowornchai et al. ${ }^{10}$ studied Thai adult mandibles and reported that the truncated type was present in $47.0 \%$ of mandibles, while the nodular, triangular, and assimilated types were present in $23.0 \%, 17.0 \%$, and $13.0 \%$ of mandibles, respectively. Jansisyanont et al. ${ }^{3}$ studied the anatomy of the ligula in dry mandibles from Thai individuals. In their study, the truncated type was the most common (46.2\%), followed by the triangular type (29.9\%), the nodular type (19.6\%), and the assimilated type (4.3\%). In contrast, in our study, the nodular type was the most common shape of the lingula (32.8\%), followed by the triangular type (31\%), the truncated type (25.9\%), and the assimilated type (10.3\%). These discrepancies reflect racial differences among the subjects in these studies.

Jansisyanont et al. ${ }^{3}$ reported that the tip of the lingula was positioned $8.2 \pm 2.3 \mathrm{~mm}$ above the occlusal plane. The location was $20.6 \pm 3.5 \mathrm{~mm}$ from the anterior border of the mandible and $16.6 \pm 2.9 \mathrm{~mm}$ from the mandibular notch. In this study, the distance of the lingula from the $\mathrm{MnF}$ was $8.8 \pm 2.6 \mathrm{~mm}$. The distance between the tip of the lingula and the occlusal plane was $8.9 \pm 5.3 \mathrm{~mm}$. The distance between the anterior border and the $\mathrm{MnF}$ was $15.5 \pm 2.6 \mathrm{~mm}$, and the distance between the posterior border of the mandible and the $\mathrm{MnF}$ was $18.1 \pm 2.2 \mathrm{~mm}$. The anteroposterior position of the lingula on the surface of mandibular ramus was almost in the central area. The distance between the gonion and the MnF was $21.0 \pm 3.9 \mathrm{~mm}$. Based on these results, the insertion point of the needle into the mouth during the IAN block technique should be approximately 9 $\mathrm{mm}$ above the occlusal plane.

There are some limitations of this study. First, the thickness of the soft tissue that the needle passes through could not be measured. The length from the anterior margin of the mandible to the $\mathrm{MnF}$ was $15.5 \pm 2.6 \mathrm{~mm}$. A short needle is $20 \mathrm{~mm}$ long. Even though the length of a short needle may be sufficient to reach the $\mathrm{MnF}$, the hub of the needle might touch the surface of the oral mucosa. The hub is the weakest point of the needle, and is a common location where needles break. ${ }^{15}$ Therefore, a long 25 - or 27 -gauge needle has been recommended for the IAN block technique. ${ }^{15,16}$ However, the thickness of the soft tissue over this area has not been studied, and further research into the proper length of the needle is needed. The distance between the anterior border of the mandibular ramus and the MnF could not be measured in some cases. In those cases, the position of the $\mathrm{MnF}$ was below the occlusal plane, and the anterior margin was merged with the alveolar bone process.

In conclusion, $\mathrm{CBCT}$ provided sufficient information on the $\mathrm{MnF}$ and the lingula. For clinicians, it is essential to be familiar with the anatomy of the $\mathrm{MnF}$ and the lingula in order to perform IAN block successfully. Knowledge of the insertion point and of the depth of needle insertion is needed to achieve sufficient anesthesia of the mandible.

\section{Acknowledgments}

This manuscript was adapted from Byeong-Seob Ahn's master's thesis.

\section{Conflicts of Interest: None}

\section{References}

1. Kamel HA, Toland J. Trigeminal nerve anatomy: illustrated using examples of abnormalities. AJR Am J Roentgenol 2001; 176: 247-51.

2. Tuli A, Choudhry R, Choudhry S, Raheja S, Agarwal S. Variation in shape of the lingula in the adult human mandible. J Anat 2000; 197: 313-7. 
3. Jansisyanont P, Apinhasmit W, Chompoopong S. Shape, height, and location of the lingula for sagittal ramus osteotomy in Thais. Clin Anat 2009; 22: 787-93.

4. Muto T, Shigeo K, Yamamoto K, Kawakami J. Computed tomography morphology of the mandibular ramus in prognathism: effect on the medial osteotomy of the sagittal split ramus osteotomy. J Oral Maxillofac Surg 2003; 61: 89-93.

5. Haas DA. Alternative mandibular nerve block techniques: a review of the Gow-Gates and Akinosi-Vazirani closed-mouth mandibular nerve block techniques. J Am Dent Assoc 2011; 142 Suppl 3: 8S-12S.

6. Pogrel MA, Bryan J, Regezi J. Nerve damage associated with inferior alveolar nerve blocks. J Am Dent Assoc 1995; 126: 1150-5.

7. Meechan JG. How to overcome failed local anaesthesia. $\mathrm{Br}$ Dent J 1999; 186: 15-20.

8. Keetley A, Moles DR. A clinical audit into the success rate of inferior alveolar nerve block analgesia in general dental practice. Prim Dent Care 2001; 8: 139-42.

9. Afsar A, Haas DA, Rossouw PE, Wood RE. Radiographic localization of mandibular anesthesia landmarks. Oral Surg Oral Med Oral Pathol Oral Radiol Endod 1998; 86: 234-41.

10. Kositbowornchai S, Siritapetawee M, Damrongrungruang T, Khongkankong W, Chatrchaiwiwatana S, Khamanarong K, et al. Shape of the lingula and its localization by panoramic radiograph versus dry mandibular measurement. Surg Radiol Anat 2007; 29: 689-94.

11. Ezoddini Ardakani F, Bahrololoumi Z, Zangouie Booshehri M, Navab Azam A, Ayatollahi F. The position of lingula as an index for inferior alveolar nerve block injection in 7-11-year-old children. J Dent Res Dent Clin Dent Prospects 2010; 4: 47-51.

12. Loubele M, Guerrero ME, Jacobs R, Suetens P, van Steenberghe D. A comparison of jaw dimensional and quality assessments of bone characteristics with cone-beam CT, spiral tomography, and multi-slice spiral CT. Int J Oral Maxillofac Implants 2007; 22: 446-54.

13. Ludlow JB, Ivanovic M. Comparative dosimetry of dental CBCT devices and 64-slice CT for oral and maxillofacial radiology. Oral Surg Oral Med Oral Pathol Oral Radiol Endod 2008; 106: 106-14.

14. Ludlow JB, Davies-Ludlow LE, Brooks SL. Dosimetry of two extraoral direct digital imaging devices: NewTom cone beam CT and Orthophos Plus DS panoramic unit. Dentomaxillofac Radiol 2003; 32: 229-34.

15. Malamed SF, Reed K, Poorsattar S. Needle breakage: incidence and prevention. Dent Clin North Am 2010; 54: 745-56.

16. Malamed SF. Is the mandibular nerve block passé? J Am Dent Assoc 2011; 142 Suppl 3: 3S-7S. 